This is a postprint version of:

Álvarez-Bornstein, B., Díaz-Faes, A. A., \& Bordons, M. (2019). What characterises

funded biomedical research? Evidence from a basic and a clinical

domain. Scientometrics, 119(2), 805-825.

The final publication is available at Springer via: https://doi.org/10.1007/s11192-019-03066-3

\title{
What characterises funded biomedical research? Evidence from a basic and a clinical domain
}

\author{
Belén Álvarez-Bornstein ${ }^{1,3}$, Adrián A. Díaz-Faes ${ }^{2}$ and María Bordons ${ }^{1}$ \\ ${ }^{1}$ belen.alvarez@cchs.csic.es; ${ }^{1}$ maria.bordons@cchs.csic.es \\ IFS, Centre for Humanities and Social Sciences (CCHS), Spanish National Research Council \\ (CSIC), Albasanz 26-28, 28037 Madrid (Spain) \\ 2 diazfaes@ingenio.upv.es \\ INGENIO (CSIC-UPV), Universitat Politécnica de València, 46022 Valencia (Spain) \\ ${ }^{3}$ Library and Information Science Department, Faculty of Library and Information Sciences, \\ Complutense University (UCM), Santísima Trinidad 37, 28010 Madrid (Spain)
}

\begin{abstract}
Improving our knowledge about funding patterns in different research domains and how funding contributes to research is a matter of great interest for funders and policymakers. This paper aims to (a) compare the funding patterns of two biomedical domains that differ in their basic vs. clinical nature, and (b) to elucidate the factors that influence the presence of funding. To do so, we draw on the scientific output of Spain-based researchers in the Virology (basic) and Cardiac and Cardiovascular Systems (clinical) domains as covered by the Web of Science database. Funding rate, public vs. private funding source, and national vs. foreign origin of the funds are examined through an analysis of funding acknowledgements in published papers. The relationship between funding and different bibliometric indicators such as impact, collaboration, basic or clinical research level, and authors' institutional sector is scrutinised. Funded studies tend to have higher impact and are more likely to have foreign partners, findings which are aligned with the objectives pursued by public funding agencies. Clinical research and research done in hospital settings are less likely to be funded. The likelihood of funding increases with the number of institutions in the clinical domain but not in the basic one. Although collaboration is fostered by public research agencies and funding may enhance the establishment of collaborative links among researchers, the likelihood of being funded does not always increase with the number of institutions, because other factors such as the type of institution and the clinical or basic nature of the research have a significant moderating effect.
\end{abstract}

\section{Introduction}

Measuring the returns of research is a matter of great concern for funding bodies, which need to demonstrate the benefits derived from their investment. Accordingly, studies of the output and outcomes arising from funded research are gaining ground in advanced economies to determine the success of funding initiatives. Because scientific publications are an essential 
output of research, bibliometric indicators have emerged as useful measures to track the results of funding streams (Campbell et al. 2011). The facts that funding acknowledgments in publications are becoming mandatory in science and that some bibliographic databases such as Web of Science (WoS) or Scopus are now collecting data on them have created new opportunities for funding organisations to track the outputs from their investment (Rigby, 2011), especially in the most internationally-oriented fields.

Studying the relationship between funding and variables such as productivity or impact can be a particularly relevant way to increase our knowledge about the effectiveness of investments in research. In this regard, it has been pointed out that funding may allow scientists to improve their infrastructures and access to data, to incorporate skilled members into their teams, and to attend conferences and strengthen collaborative links with colleagues. Together, these opportunities may have a positive influence on research. Moreover, on the assumption that funding is usually awarded through a review process, higher quality could be expected for funded research, since it has successfully undergone peer review (Lewison and Dawson, 1998). As stated by Wang and Shapira $(2015$, p. 3), "peer review processes aim to filter out weaker research proposals and reward more promising research ideas". Although being funded also entails certain burdens such as the administrative tasks associated with managing the funds awarded, which reduce the time available for research (Decker et al. 2011; Wang and Shapira, 2015), a positive final balance is usually expected. The relationship between funding and scientific output has been analysed in a number of studies centred on specific institutions (Sandstrom, 2009; Hottenrott and Lawson, 2013), disciplines (Beaudry and Allaoui, 2012) and research programmes or funding sources (Jacob and Lefgren, 2011; Langfeldt, Bloch and Sivertsen, 2015; Ebadi and Schiffauerova, 2016; Neufeld, 2016). A positive relationship between funding and number of publications has been observed for scientists in several universities in Sweden (Sandstrom, 2009) and in the United Kingdom (Hottenrott and Lawson, 2017). Moreover, differences between the effects of public and private funding have been suggested elsewhere (Carayol and Matt, 2006; Beaudry and Allaou, 2012; Hottenrott and Lawson, 2017). Regarding research programmes, the literature shows a positive relationship between funding and scientists' productivity (Schneider and van Leeuwen, 2014), even though the positive effect is sometimes very limited (Jacob and Lefgren, 2011; Neufeld, 2016), and no clear differences between achievement before and after receiving funding has been observed in other studies (Bol, de Vaan and van de Rijt, 2018).

Concerning the effects of funding on the impact of research, some studies have focused on specific funding instruments or agencies to assess differences between funded and nonfunded scientists (Campbell et al. 2010), while others have analysed differences in impact before and after the funding decision (Langfeldt, Bloch and Sivertsen, 2015). Thus, higher citation rates were found for funded research compared to non-supported research at the National Cancer Institute of Canada (Campbell et al. 2010) and the National Institutes of Health in the USA (Boyack and Jordan, 2011). However, although funding increased the share of highly cited papers, it had no effect on the average citation rate of funded scientists in a study of the effects of research grants in Denmark and Norway, where funding was reported to have a greater effect on productivity than on impact (Landgfeldt, Bloch and Sivertsen, 2015). Interestingly, the amount of funding also appears to be more strongly correlated with productivity than with research impact (Godin, 2003). In a study on scientists funded by a research granting agency in Canada, impact was mildly positively correlated to funding, but the highest impact articles do not clearly flow from the largest amount of funding (Fortin and Currie, 2013). These findings notwithstanding, it should be borne in mind that it is difficult to isolate the output of a single grant or funding source because many scientists hold multiple grants concurrently. As a result, many studies yield inconclusive results. 
Studies of the effects of funding on research impact at other levels of aggregation such as journals, disciplines or countries have yielded diverging results. No clear relationship was observed between funding and citations received by publications in some studies focused on specific journals (Harter and Hooten, 1990; De Granda-Orive et al., 2015; Rigby, 2011) and research topics (Pao, 1991; Haslam et al. 2008). However, grant-funded publications attained higher impact than non-funded publications in a number of disciplines such as economics (Peritz, 1990), gastroenterology (Lewison and Dawson, 1998), library and information science (Zhao, 2010), and nanotechnology (Wang and Shapira, 2015). Funding was also positively associated with citation impact in a study of several fields in six European countries (Gök et al. 2016), where citation impact was also positively associated with funding variety. The inconsistent results across studies may be due to differences among fields, countries, and sizes of the data sets, as well as to the number and type of influencing factors considered.

Funding may have a positive effect on scientific output because it provides access to research resources, but also because it fosters collaboration. Previous studies have shown a positive relationship between financial support and collaboration (Heffner, 1981; Bozeman and Corley, 2004; Ubfal and Maffioli, 2011; Clark and Llorens, 2012; Astrom et al. 2013; Yegros and Costas, 2013). This finding is not surprising, since grants usually support teams rather than individual scientists, and empirical evidence of the positive influence of funding on collaboration has also been reported elsewhere (e.g. De Fazio, Lockett and Wright, 2009; Ubfal and Maffioli, 2011). Public funding policies are generally designed to foster research collaboration and the creation of research networks, on the assumption that collaboration helps to increase research productivity and thereby favours scientific advances (see e.g. Katz and Martin, 1997; Lariviere et al. 2015). Collaboration may improve research quality by involving researchers from different backgrounds, thereby providing group synergies that allow joint work to lead to more creative and relevant results. As an example of this pathway to enhanced scientific output, collaborative research tends to be published in higher-quality journals and be cited more frequently (Wuchty, Jones and Uzzi, 2007; Bordons, Aparicio and Costas, 2013).

The relationships summarised above thus exemplify how funding may have indirect positive effects on scientific output and impact by stimulating scientific collaboration (Ebadi and Schiffauerova, 2016). In a survey of academic scientists in different science fields in the USA, Lee and Bozeman found evidence of a positive effect of grants on both productivity and collaboration (2005). In the context of European Union-funded research networks, funding was reported to have a more significant direct influence on researcher productivity than collaboration (Defazio, Lockett and Wright, 2009). More recently, Yan et al. (2018) observed that multiple authorship magnifies the association between funding and citations in a selection of highly cited journals in seven disciplines. The extent to which the positive effects of funding on scientific output are secondary to increased collaboration is an issue that deserves more detailed scrutiny, with a special focus on potential differences between disciplines given that research strategies, collaboration practices and funding effects may vary across fields.

This article aims at improving our knowledge of the main features of funded research compared to non-funded research in two biomedical domains, and to investigate the relationship between funding and the research impact of publications. We have focused here on biomedicine because of its important social impact, inasmuch as it contributes to improvements in health and quality of life at the population-wide level. We selected one clinical and one basic domain for comparison, because differences may exist in research and collaborative practices as well as in how funding affects research performance depending on the clinical or basic nature of the research (Clark and Llorens, 2012). Moreover, it is clear that current research in the life sciences relies heavily on funding because of the increasing need to deal with complex interdisciplinary problems, obtain access to cutting-edge equipment, and 
bring together scientists from different specialities and settings (e.g. universities, hospitals, public and private research organisations). This situation, together with the tight constraints on public budgets, has led to radical changes in funding structures and practices, such as the rise in prominence of the role played by research consortia, third-party funded research projects, and international collaborative research networks (Müller, 2012).

\section{Objectives}

The objectives of this study are two-fold: a) to analyse the scientific output of Spain in two biomedical domains and describe their funding profile through an analysis of funding acknowledgement data in the WoS, and b) to characterise funded research, as opposed to non-funded research, and to identify which aspects of the scientific endeavour contribute to the presence of funding, with particular attention to the features of impact and collaboration, and to differences between domains. We wished to answer the following questions: Are there differences between domains in funding rates and types of funding? Is there a positive relationship between funding and research impact? Is funding associated with higher levels of collaboration? Do these relationships vary depending on the basic or clinical nature of the field? The findings of the present study will increase our understanding of the role of funding in the creation of new knowledge, and will provide useful information to guide future funding decisions.

\section{Methods}

Scientific articles by authors based in Spain and published during 2010-2014 were selected from WoS in two biomedical domains: Cardiac and Cardiovascular Systems (CARD), which is clinically-oriented, and Virology (VIROL), which is a mainly basic-science domain. The delimitation of both domains was based on the WoS classification of journals into subject categories. Only citable items (articles and reviews) published in English were considered, since funding acknowledgements are regularly covered by WoS only for items published in this language (Alvarez-Bornstein et al., 2017). A total of 4,015 publications were included in the analysis.

To capture the funding profile of biomedical research in Spain, we draw on information included in the WoS funding acknowledgement (FA) field, which has been used in this database since August 2008. The Funding Agency section, which contains the names of the agencies that supported the research, was normalised and classified with a web application created for this purpose. In addition, a master file of agencies was built to compile, for each agency, its full-normalised name, acronym (if any), institutional sector (see below), type of funding (public, private, mixed) and country.

For data analysis, we used two approaches. First, we conducted a comparative analysis of the two domains on the basis of their scientific output and funding profile. Then we used regression analysis to identify which aspects of the scientific endeavour contributed to the presence of funding acknowledgments, and to more fully characterise funded research.

\section{Comparative analysis of the two domains}

a) Scientific output. First, the domains were characterised according to the involvement of different institutional sectors in the research. The institutional sector of the authors' affiliations was considered, and six different sectors were distinguished: higher education, hospital, government, business, non-profit organisation (NPO) and multi-sector institution. 
It should be noted that national research centres such as the Spanish National Research Council (CSIC) and other public research organisations are included in the government sector. Multi-sector institutions include entities that operate through the joint efforts of public and private organisations.

Second, the two domains were characterised regarding their activity, collaboration and research impact. Specifically, the following indicators were obtained: number of articles, collaboration rate, average number of authors per article, average number of institutions per article, citation rate relative to the world average (RCR), share of highly cited papers (HCP) (share of papers among the most cited $10 \%$ worldwide), and share of papers in top journals by impact factor, i.e. Q1 (first quartile) and D1 (first decile). Citations from the publication year to 2015 were considered.

Finally, as a proxy for the basic or clinical nature of the research, we attempted to use the $\mathrm{CHI}$ research level of the journal as described by Narin et al. (1976), which range from $1-$ most applied - to 4 -most basic $-(\mathrm{RL1}=$ clinical observation; $\mathrm{RL2}=$ clinical mix; $\mathrm{RL3}=$ clinical investigation; RL4 = basic research). However, this classification is not up to date, so no level was assigned to the journals included most recently in WoS. Specifically, about $45 \%$ of CARD papers and $20 \%$ of VIROL papers were published in journals with an unknown level, so we discarded this indicator. Instead, the research level described by Boyack et al. (2014) was used. In Boyack's classification, the probability of belonging to each research level is obtained for each paper taking into account the title and abstract words as well as the distribution of cited references by research levels. Then, each article is classified in the single level for which it had the highest probability. This indicator has the advantage of classifying individual articles instead of journals. It was available for $96 \%$ of the papers.

b) Funding pattern. Considering data included in the FA field, the following indicators were obtained for the two domains:

- Funding rate: percentage of papers that include funding acknowledgment data.

- Average number of funding agencies per article.

- Percentage distribution of papers by institutional sector of the funders. Five different sectors were considered: higher education, central government, regional government, business, and private-non-profit institution. We distinguished between central and regional governments because both distribute funds for research in Spain and have their own research plans and programmes.

- Percentage distribution of papers by nationality of funders. First, Spanish and foreign funders were distinguished; second, the nationality of foreign funders was identified; third, the share of papers with supranational funders such as international organizations, or specifically the European Commission, was calculated.

- Percentage distribution of papers by type of funding agency. We distinguish between public and private funding agencies.

\section{Regression analysis}

Because funding acknowledgement is a binary characteristic (i.e. papers include or do not include a mention of funding), logistic regression analysis was conducted with funding acknowledgement as the dependent variable. Various independent variables were considered to provide information about different aspects of the research:

- Research impact. To elucidate the association between impact and funded research, we drew on size-independent indicators that account for the individual impact of research articles and for the relative prestige of journals where authors have published their 
research. Specifically, the following indicators were used: RCR, HCP and share of papers in Q1 journals. RCR was categorised in a way that allowed the effects to be tested by thresholds ( 1 = citation rate lower than world average, $2=$ equal to or above the world average but below highly cited papers, 3 = highly cited papers).

- Collaborative research patterns. Because funding policies aim to promote teamwork and research collaboration to achieve research goals, the following indicators were calculated: number of authors per article, number of institutions per article, and international collaboration $(1=$ Yes, $0=\mathrm{No})$. The number of authors was removed from the analysis due to multicollinearity problems. In accordance with a rationale similar to that used for the RCR, three categories were considered for the number of institutions (1 institution, 2-4 institutions, and more than 4).

- Basic vs. clinical orientation of the research. Given that the need for research to be funded as well as collaboration practices within and between domains may strongly rely on domain nature and scope (fundamental research on the causes of diseases vs. research aimed at improving clinical practice), the research level of papers was considered to differentiate between basic and clinical research. The four categories used for research level were aggregated into two broad classes: clinical (levels 1 and 2) and basic (levels 3 and 4).

- Domain. This was included as a variable to explore differences between the two domains analysed. Virology was the reference category in the regression model.

- Institutional sector. This was introduced as a control variable, because the type of institutional setting in which researchers work might affect the presence of funding. In other words, the tendency of scientists to seek economic support or their subsequent success in obtaining it may differ depending on the institutional sector of the authors' affiliations. To account for this, six non-mutually exclusive binary variables coded according to the authors' specific affiliation were considered. For example, the variable "University_address" was assigned a value 1 if any of the authors were affiliated with a university, and was given a value of 0 otherwise.

Table 1 summarises the distribution of variables. Several models were obtained by adding different variables to the model. Multicollinearity problems were ruled out by examining tolerance and variance inflation factor (VIF) values. Nagelkerke's pseudo $R^{2}$ was calculated as a measure of the proportion of variance explained by each model. All statistical analyses were done with SPSS v. 25 software. 
Table 1. Descriptive statistics

\begin{tabular}{llrrrr}
\hline Variable & Category & CARD & \multicolumn{3}{c}{ VIROL } \\
\cline { 3 - 5 } & & No. & $\%$ & No. & $\%$. \\
\hline Funding & No & 1180 & 42.9 & 127 & 10.1 \\
& Yes & 1572 & 57.1 & 1136 & 89.9 \\
Research level & Clinical (1+2) & 2283 & 87.6 & 406 & 32.4 \\
No. institutions & Basic (3+4) & 323 & 12.4 & 847 & 67.6 \\
& 1 institution & 393 & 14.3 & 173 & 13.7 \\
International & $2-4$ institutions & 1125 & 40.9 & 657 & 52.0 \\
collaboration & $>4$ institutions & 1234 & 44.8 & 433 & 34.3 \\
First-quartile journal & No & 1400 & 50.9 & 641 & 50.8 \\
& Yes & 1352 & 49.1 & 622 & 49.2 \\
RCR & No & 1398 & 52.4 & 658 & 52.3 \\
& Yes & 1270 & 47.6 & 600 & 47.7 \\
& RCR $<1$ (low) & 1680 & 61.0 & 795 & 62.9 \\
& RCR $\geq 1$ and $<$ HCP (medium) & 557 & 20.2 & 307 & 24.3 \\
& HCP (high) & 515 & 18.7 & 161 & 12.7 \\
\hline
\end{tabular}

Notes: $\mathrm{RCR}=$ Relative citation rate. $\mathrm{HCP}=$ Highly cited paper

The reference category was the first one for all variables.

Finally, it should be noted that the expression "funded papers" is used in this article to refer to those papers which included any explicit acknowledgement to one or more funding sources in the WoS FA field, while the expression "non-funded papers" identifies those with no relevant information in the FA field.

\section{Results}

\section{Research output and funding by domain}

In the period from 2010 to 2014, a total of 2,752 articles in CARD and 1,263 in VIROL were published. CARD was more clinically oriented than VIROL, as shown by its lower research level (1.9 vs. 3.1). Moreover, around $84 \%$ of CARD articles were published by scientists located at hospitals, whereas government institutions, and to a lesser extent, hospitals, were the most frequent affiliations of VIROL authors ( $48 \%$ and $41 \%$, respectively) (Figure 1 ). Both domains displayed similar collaboration patterns, with more than one institution in approximately $86 \%$ of the papers, and international partners in half of the articles, while team size, measured as the average number of authors per paper, was slightly larger in CARD (9.7 vs. 8.5). The CARD domain showed higher international impact as observed by its greater RCR (1.4 vs. 1.1), higher percentage of articles in D1 journals (20.3 vs. 13.3), and larger share of HCP articles among the $10 \%$ most cited worldwide (18.7 vs. 12.7) (Appendix 1). 


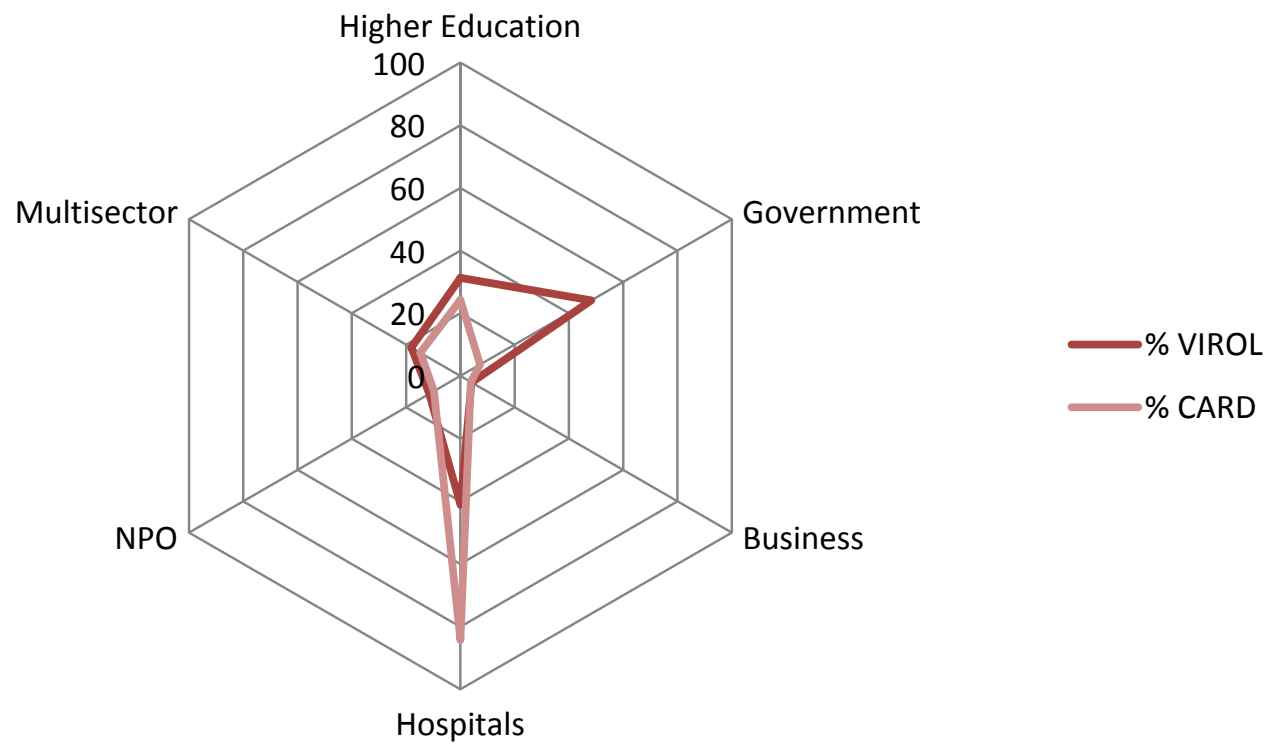

Figure 1. Scientific output of CARD and VIROL by institutional sector of authors' affiliations

Funding was more frequently acknowledged in VIROL (89.9\% of the articles) than in CARD (57.1\%). The average number of funders per paper was 3.8 in both domains, but approximately $33 \%$ of papers in CARD mentioned only one funder vs. $18 \%$ in VIROL, and the most frequent value (mode) was 1 funder per paper in CARD vs. 3 funders per paper in VIROL (Figure 2). The main research funders in both domains were the Carlos III Institute of Health (ISCIII) and the Ministry of Economy and Competitiveness in Spain.

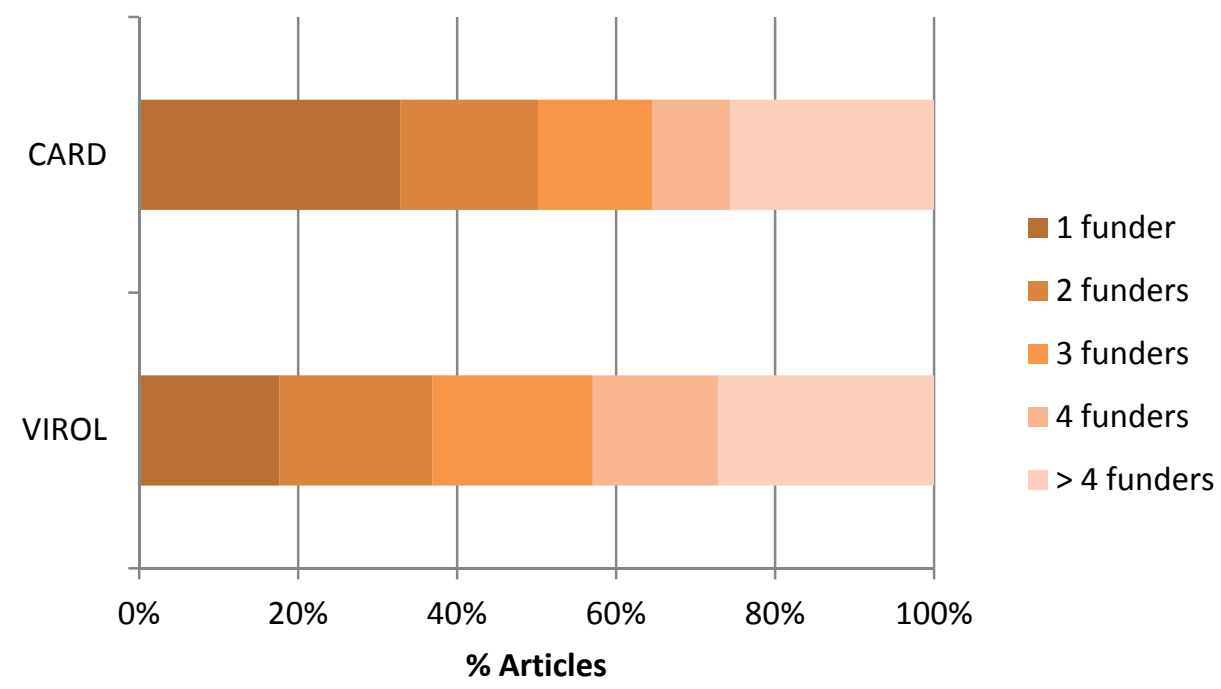

Figure 2. Distribution of articles by number of funders per article.

Concerning the institutional sector of funders, the differences between the two domains are shown in Table 2. The central government was the largest financial contributor in both domains and was responsible for almost all funding in VIROL $161.8 \%$ in CARD vs. $91.1 \%$ in 
VIROL), whereas the business sector was more active in CARD (45.3\% vs. $16.8 \%$ ). Regarding the public or private nature of funding, a notably higher share of papers received private support in CARD (65\% vs. $42 \%)$ although public support predominated in both domains. In addition, two thirds of the articles in both domains reported funding from foreign countries. European Union funds (especially the European Regional Development Fund [ERDF] and the European Framework Programmes for research and technological development) were acknowledged in $14.5 \%$ of the papers in CARD vs. $33.6 \%$ in VIROL.

Table 2. Funding profiles of the CARD and VIROL domains. Distribution of papers by institutional sector, type of funding and nationality of funding agencies

\begin{tabular}{lcccc}
\hline & \multicolumn{2}{c}{ CARD (n=1,572) } & \multicolumn{2}{c}{$\begin{array}{c}\text { VIROL (n=1,136) } \\
\text { No. }\end{array}$} \\
& $\begin{array}{c}\text { No. } \\
\text { articles }\end{array}$ & $\%$ & 115 & articles \\
\hline $\begin{array}{l}\text { Institutional sector of funding agencies } \\
\quad \text { Higher education }\end{array}$ & 208 & 13.3 & 10.2 \\
$\quad$ Central government & 969 & 61.8 & 1027 & 91.1 \\
Regional government & 347 & 22.1 & 345 & 30.6 \\
NPO & 486 & 31.0 & 306 & 27.2 \\
$\quad$ Business & 711 & 45.3 & 189 & 16.8 \\
Type of funding & & & & \\
$\quad$ Only public & 543 & 34.6 & 657 & 58.3 \\
$\quad$ Only private & 501 & 31.9 & 68 & 6.0 \\
$\quad$ Public and private & 525 & 33.5 & 402 & 35.7 \\
Nationality of funding agencies & & & & \\
$\quad$ Only Spain & 491 & 31.3 & 381 & 33.8 \\
$\quad$ Only foreign countries & 648 & 41.3 & 235 & 20.9 \\
$\quad$ Spain and foreign & 373 & 23.8 & 505 & 44.8 \\
$\quad$ Not assigned & 57 & 3.6 & 6 & 0.5 \\
\hline
\end{tabular}

Notes: NPO=Non-profit organisations. Percentages computed with reference to the total number of papers in each domain. The sum of the percentages exceeds $100 \%$ because some articles acknowledged more than one agency.

\section{Factors explaining the presence of financial support}

To investigate the main drivers of funded research and explore a range of factors which might influence the presence of funding a logistic regression was applied. The main results are summarised in Table 3. 
Table 3. Results of the logistic regression model to identify drivers of funding

\begin{tabular}{|c|c|c|c|c|c|c|c|c|c|}
\hline & \multicolumn{3}{|c|}{ Model 1 (Baseline model) } & \multicolumn{3}{|c|}{ Model 2 (Domain*RCR) } & \multicolumn{3}{|c|}{$\begin{array}{c}\text { Model } 3 \\
\text { (Domain*No. institutions) }\end{array}$} \\
\hline & B & Wald & OR & B & Wald & OR & B & Wald & OR \\
\hline First quartile & $0.537^{* * *}$ & 36.394 & 1.711 & $0.539 * * *$ & 36.671 & 1.714 & $0.539 * * *$ & 36.454 & 1.715 \\
\hline RCR & & 9.274 & & & 0.057 & & & 8.042 & \\
\hline Medium & $0.268^{*}$ & 6.097 & 1.308 & $0.060^{*}$ & 0.055 & 1.062 & $0.259 * *$ & 5.640 & 1.296 \\
\hline $\begin{array}{l}\text { High } \\
\text { (reference=Low) }\end{array}$ & $0.308^{* *}$ & 5.814 & 1.360 & $-0.001 * * *$ & 0.000 & 0.999 & $0.277^{*}$ & 4.688 & 1.319 \\
\hline No. institutions & & 48.375 & & & 47.732 & & & 12.250 & \\
\hline 2-4 institutions & 0.132 & 0.945 & 1.141 & 0.125 & 0.855 & 1.134 & $-1.164 * *$ & 9.476 & 0.312 \\
\hline $\begin{array}{l}>4 \text { institutions } \\
\text { (reference=1 inst.) }\end{array}$ & $0.815^{* * *}$ & 25.740 & 2.260 & $0.806^{* * *}$ & 25.069 & 2.239 & -0.679 & 2.792 & 0.507 \\
\hline International collab. & $0.364^{* * *}$ & 11.110 & 1.439 & $0.363^{* * *}$ & 11.069 & 1.438 & $0.369 * * *$ & 11.600 & 1.446 \\
\hline Basic research level & $0.823 * * *$ & 35.442 & 2.278 & $0.816^{* * *}$ & 34.766 & 2.261 & $0.805^{* * *}$ & 33.947 & 2.236 \\
\hline CARD & $-1.273^{* * *}$ & 103.384 & 0.280 & $-1.361^{* * *}$ & 87.586 & 0.256 & $-2.713^{* * *}$ & 51.109 & 0.066 \\
\hline CARD*RCR & & & & & 1.569 & & & & \\
\hline CARD*Medium RCR & & & & 0.251 & 0.804 & 1.285 & & & \\
\hline CARD*High RCR & & & & 0.355 & 1.043 & 1.426 & & & \\
\hline CARD*No. institutions & & & & & & & & 18.145 & \\
\hline CARD*2-4 instit. & & & & & & & $1.553^{* * *}$ & 15.146 & 4.725 \\
\hline CARD*>4 instit. & & & & & & & $1.763^{* * *}$ & 17.568 & 5.829 \\
\hline University_address & $0.701 * * *$ & 39.948 & 2.016 & $0.702^{* * *}$ & 39.986 & 2.019 & $0.703 * * *$ & 40.428 & 2.021 \\
\hline Government_address & $0.629 * * *$ & 15.224 & 1.875 & $0.629 * * *$ & 15.226 & 1.876 & $0.598 * * *$ & 13.853 & 1.819 \\
\hline Business_address & 0.120 & 0.280 & 1.127 & 0.122 & 0.291 & 1.130 & 0.126 & 0.316 & 1.134 \\
\hline Hospital_address & $-0.396 * *$ & 7.799 & 0.673 & $-0.396^{* *}$ & 7.783 & 0.673 & $-0.328^{*}$ & 5.331 & 0.720 \\
\hline NPO_address & $0.320^{*}$ & 4.142 & 1.377 & $0.321^{*}$ & 4.168 & 1.379 & $0.343^{*}$ & 4.757 & 1.409 \\
\hline Multisectoral_address & $0.931 * * *$ & 43.804 & 2.536 & $0.931 * * *$ & 43.803 & 2.537 & $0.935^{* * *}$ & 44.713 & 2.548 \\
\hline Constant & $0.594^{* * *}$ & 9.529 & 1.811 & $0.671^{* * *}$ & 10.874 & 1.957 & $1.751^{* * *}$ & 22.120 & 5.761 \\
\hline Nagelkerke's $R^{2}$ & & 0.342 & & & 0.343 & & & 0.349 & \\
\hline
\end{tabular}

Model 1 includes the main effects of the different explanatory variables on funding without considering interactions between variables. Positive associations were seen between funding and the variables first quartile $(\beta=0.537, P<0.001$ ), RCR (medium $\beta=0.268, P<0.05$; high $\beta=$ $0.308, P<0.01)$, number of institutions ( $<4$ institutions $\beta=0.815, P<0.001)$ and international collaboration ( $\beta=0.364, P<0.001$ ), as shown by the significant positive $\beta$ coefficients. The odds ratio $(O R)$ for these variables were greater than 1 , indicating that as the numerical value of each variable increased, so did the odds that funding would be reported. In contrast, papers from hospitals were less likely to report funding (OR less than 1). Papers with a multisectoral address had the greatest probability of being funded among the different institutional settings considered here (i.e., the OR for this association was the highest). Interestingly, an FA was present 2.27 times as often in basic papers as in clinical ones. Finally, an FA was present in $72 \%$ fewer CARD papers than in VIROL ones $(O R=0.28)$.

To explore whether the effect of the explanatory variables was the same in both domains, we looked for statistical interactions between domain and the remaining variables. Our main 
interest was the possible interaction between scientific domain and the variables that measured impact and collaboration (Table 3). Model 2 thus considered the interplay between research impact (RCR) and scientific domain. The results were consistent with the previous model, although the interplay was not significant -suggesting that the effect of paper impact on funding presence did not vary significantly by domain. The interaction between domain and first quartile was also non-significant (not shown).

In contrast, a significant interaction was found for domain and number of institutions in Model 3 ; in other words, the effect of the number of institutions differed depending on the field. A striking negative relationship was seen between number of institutions and funding in VIROL, where single-institution papers were the most likely to be funded. In contrast, the number of institutions was positively associated with funding in CARD, since articles with 2 to 4 institutions and those with more than 4 institutions were, respectively, 1.47 ( $\beta$ 1.553-1.164 = $0.389 ; \operatorname{Exp}(0.389)=1.47)$ and 2.9 times $(\beta 1.763-0.679=1.084 ; \operatorname{Exp}(1.084)=2.9)$ more likely to note funding than those with only 1 institution. Model 3 correctly classified approximately $78 \%$ of the cases, and Nalgelkerke's pseudo $R^{2}$ showed that about $35 \%$ of the variation was explained by the model.

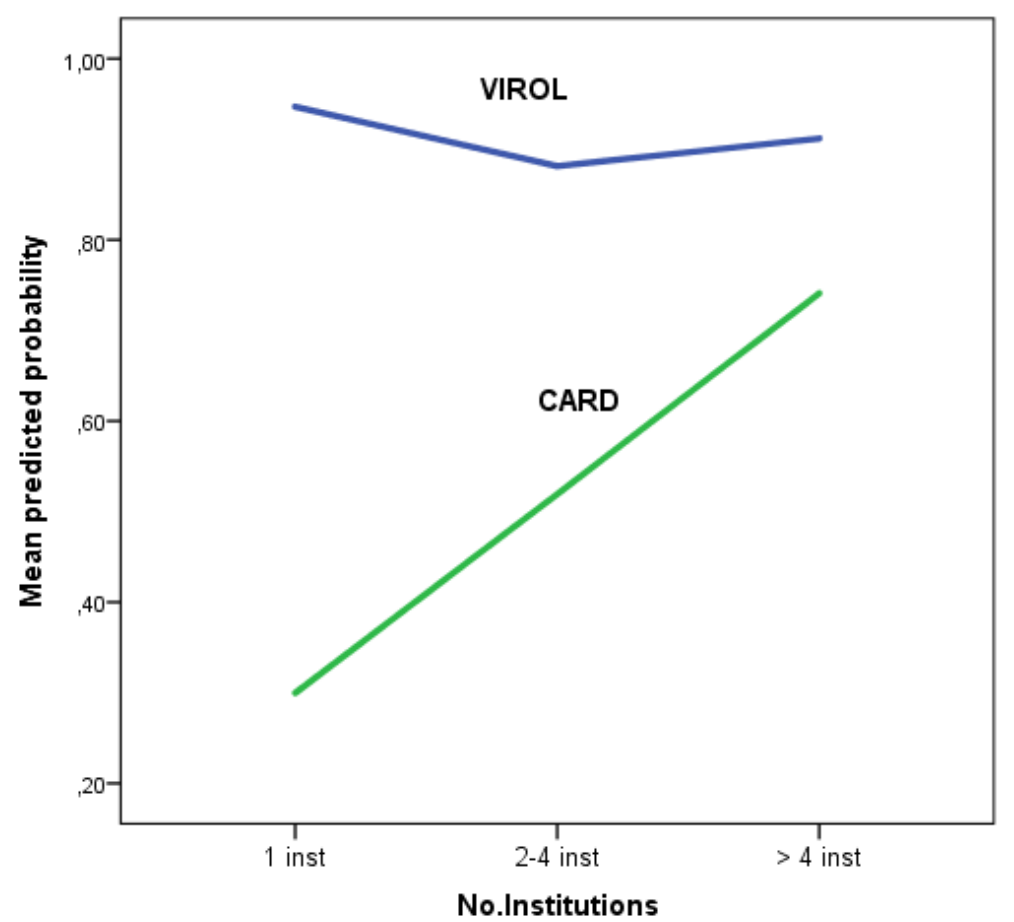

Figure 3. Predicted probabilities for funding rate by domain and number of institutions per article (Model 3)

The interaction between domain and number of institutions is plotted in Figure 3. The funding rate in VIROL was higher than in CARD regardless of the number of institutions. However, the lines are not parallel, and have different slopes, because the effect of the number of institutions was not the same in both domains. The slope for CARD is steeper than for VIROL, indicating in the former that differences in funding were larger depending on the number of institutions per article. Funding rate increased for CARD together with the number of institutions, whereas single-institution papers showed the highest funding rate in VIROL.

Because differences in funding rate between basic and clinical research were observed in Table 3 , the influence of research level was compared in Figure 4; the plots illustrate differences 
between domains. Firstly, we observed a higher funding rate in basic than clinical research in the two domains. Secondly, the general patterns of funding rates in Figure 3 (increased funding as the number of institutions increased in CARD vs. decreased funding in VIROL) were again seen for basic and clinical research in both domains. However, the difference in FA between basic and clinical research was greater in CARD, in particular for papers from a single institution: average funding rate ranged from around $20 \%$ in clinical papers to $80 \%$ in basic ones. Thirdly, it should be noted that papers by scientists affiliated with a single institution in VIROL had the highest funding rates for either basic or clinical research.

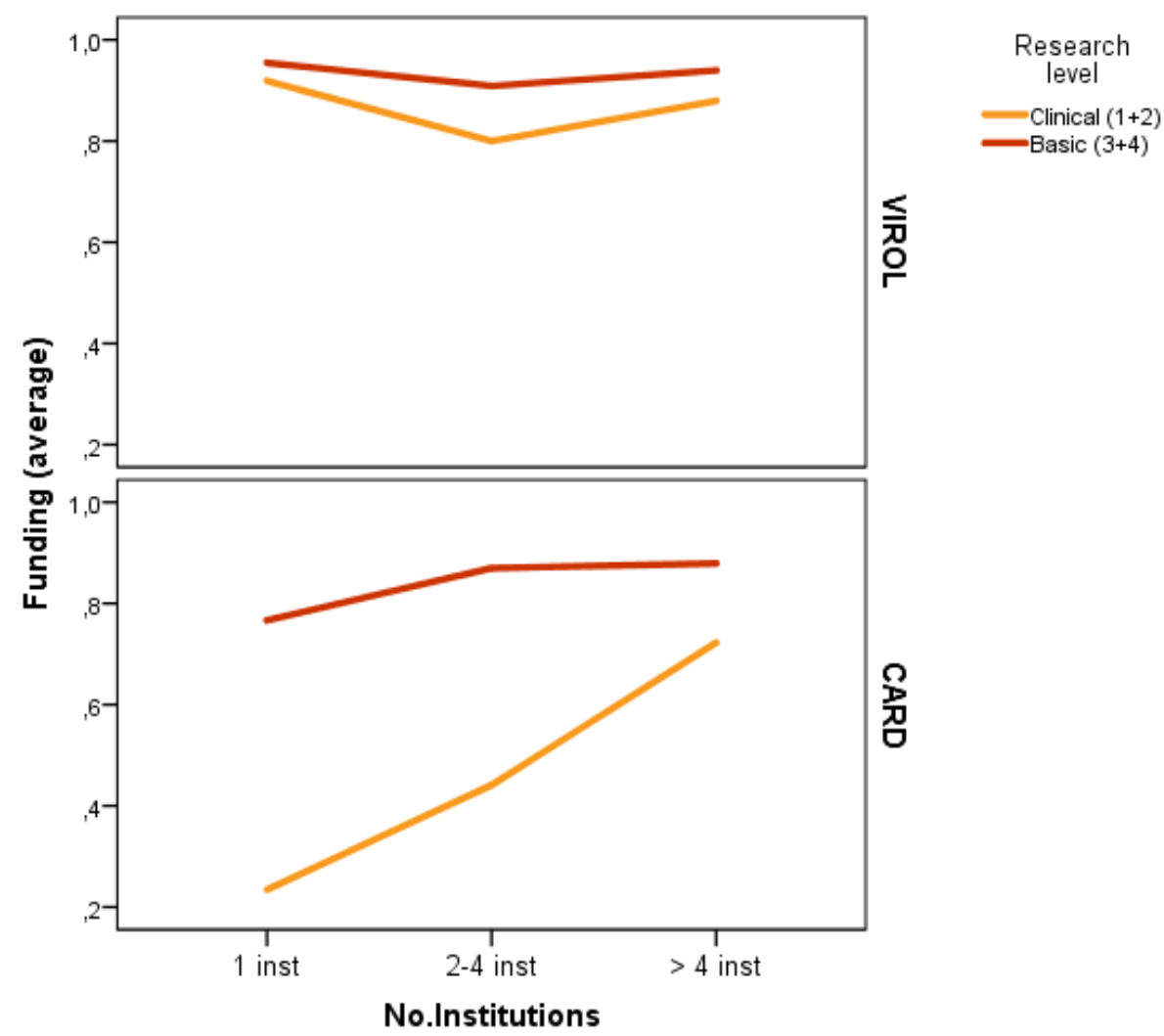

Figure 4. Predicted probabilities for funding rate by domain, number of institutions per article and research level

Overall, the lower FA rate in CARD than in VIROL can be partly explained by the higher share of papers from hospitals in the former domain, given that hospital-affililated articles were less likely to report funding. As the number of institutions increased in CARD, so did contributions from institutional sectors other than hospitals, and consequently the likelihood that funding was acknowledged. In VIROL, a large share of papers were from public research organisations, which are highly dependent on funding. Differences between basic and clinical research were smaller in VIROL regardless of the number of institutions, because clinical research in this domain is "less applied" (higher average research level, 1.94 vs. 1.65), and authors with a hospital affiliation are involved less often. As an example, we note that hospitals contributed to approximately $90 \%$ of single-institution papers in CARD, vs. $18 \%$ in VIROL.

Finally, two additional logistic regression analyses were run to explore potential differences in the likelihood that private and public funding will contribute to high impact research (publications in Q1 journals and HCP were used as dependent variables). Results reveal no differences between public and private funded papers in VIROL, the most basic field. 
Concerning CARD, papers funded only by private funders were less likely to be published in Q1 journals than those with only public funders, but they were 2 times more likely to become $\mathrm{HCP}$, which points to the final relevance of private funded research (Appendix 2).

\section{Discussion and conclusions}

This study analyses funding acknowledgements in articles published by Spain-based researchers in two biomedical domains, CARD and VIROL, as covered by the WoS database. Clear differences emerge from our data in the rate of funding acknowledgments reported, as well as in the type of funding sources regarding their national/foreign and public/private nature (Table 2). Higher rates of funding are observed in VIROL (close to 90\%), a predominantly basic field, than in CARD (57\%), a more clinically-oriented field where hospitals are frequently involved in research (Figure 1). Unsurprisingly given the large share of R\&D expenditure from the public sector in Spain (COTEC, 2018), this sector plays a key role as an external funding source in both domains, but especially in VIROL, where it is acknowledged in almost $95 \%$ of the funded papers. Interestingly, private sources are acknowledged in these domains in a large share of papers (more than 40\%), especially in CARD (65\%), due to the involvement of a research-intensive pharmaceutical industry in the biomedical sector (European Commission, 2016). National and especially multinational pharmaceutical companies are important promoters of research at hospitals, mainly through their support of clinical trials, which may provide critical medical knowledge (Butler et al. 2015).

\section{Basic research is more likely to be funded}

Our results reveal a higher funding rate for basic research, which in this study comprises papers categorised in research levels 3 or 4 (Boyack and Jordan, 2011). Not only is a higher funding rate observed in VIROL, a more basic domain than CARD, but in addition, basic papers in both domains are more likely to be funded than clinical ones, especially in CARD (Table 3). This finding is in line with the lower funding rate observed for most applied fields in a previous study (Costas and van Leeuwen, 2012), in which the authors argued that funding in these disciplines might more often come from sources that do not require explicit acknowledgement.

Closely related to this finding is the observation that in both domains, papers authored by researchers at hospitals, which are the main producers of clinical research, include funding acknowledgements significantly less often than papers by authors with other affiliations, such as higher education or government organizations - as also observed by Lewison (2003) in his study of cancer research. In fact, research at hospitals may enjoy higher levels of internal funding which is not always acknowledged by authors. Moreover, since clinical research is funded to a large extent by private companies, the fact that authors might fail to acknowledge it due to possible conflicts of interest cannot be ignored, although authors are increasingly required to disclose their financial relationships with industry (ICMJE, 2017). Finally, it should be noted that some publications arising from research in hospital settings may derive from clinical practice and not rely on a specific research project. Examples of this scenario include studies dealing with the epidemiology of diseases, effectiveness and safety of diagnostic or therapeutic techniques, and updates or reviews of preventive or therapeutic measures in specific diseases. In summary, at hospitals research carried out in the course of externallyfunded research projects, which are presumably acknowledged in publications, coexist with other research derived from clinical practice, funded internally, or funded by sources that do not require explicit acknowledgement. 


\section{Funding is associated with higher impact of research}

In this study, funded research is more likely to be published in top journals than non-funded research, and the former also receives more citations (Table 3, Appendix 1), which suggests that funders have succeeded in obtaining a positive return on their investment in the form of high quality and influential research. Nothwithstanding, we are aware that besides the scientific impact, biomedical research may also have an important impact on society, as far as it contributes to enhance population health, but the analysis of this dimension of the research is beyond the objectives of this paper.

Since public and private funding may differ in a number of aspects such as their objectives and allocation mechanisms, a different level of commitment with high scientific impact goals might exist. In other words, a stronger relationship between funding and Q1 publications could be expected in the case of public funding, which more clearly encourage scientific excellence and curiosity-driven research, as compared to private funded papers (most of them supported by industry), which tends to focus on more applied objectives. Our results reveal that in the case of VIROL there are not significant differences between public and private funded papers in their likelihood to attain top journals or become HCP, probably because VIROL is a very basic field where public funding predominates. However, some differences are observed in CARD, where private funded papers are less likely to be published in top journals, probably because getting published in Q1 journals is not the top priority for industry. In spite of this, private funded papers are more likely than those with only public support to become HCP, which denotes the relevance of private funded research, in particular that developed in the context of highly collaborative projects with foreign partners and support from multinational firms (Appendix 2).

It is interesting to note that funded research is more likely to involve international collaboration, increasingly promoted by public funding agencies (Wang and Shapira, 2015), and which in itself might contribute to boost the impact of research. Collaboration with foreign partners may lead to higher impact through different mechanisms widely described in the literature, such as the inclusion of a greater variety of points of view which may lead to more creativity and innovation (Reagans and Zuckerman, 2001). Another potential mechanism behind this greater impact is the enhanced dissemination of research through the authors' personal networks, which leads to increased public awareness and subsequently higher citation rates (Goldfinch et al. 2003). Interestingly, our results show that the higher impact of funded research is not only a consequence of greater international collaboration, since a positive association between funding and the share of articles in Q1 journals or the RCR is observed after different collaboration variables are controlled for, e.g., international collaboration and the number of institutions (Table 3).

We are aware that our discussion here may constitute a circular argument: Does funding help to enhance the quality and impact of the research? Or given the competitive nature of funding policies, is funding allocated to the "best" projects and researchers? Does funding foster scientific international collaboration? Or is funding allocated to the most collaborative teams, given that collaboration is viewed favourably by funding agencies? Whatever the case, our results suggest that funding contributes to high-quality research, either by preferentially funding scientists who were already top performers or by supporting "average" scientists who take advantage of the resources to carry out high-quality research. A study at more granular levels of aggregation would be needed to discriminate between these two situations, for example through an analysis of scientists' research performance that takes into account their productivity, impact and collaboration before and after receiving specific research funds. 


\section{The likelihood of being funded does not always increase with collaboration}

Theoretically, one would expect to find a positive relationship between funding rate and collaboration (Wang and Shapira, 2011; Zhao 2010). One likely explanation is that larger teams are more likely to be funded under the assumption that they are more competitive and may attain greater achievements in research. In addition, funding enhances collaboration, either through research contracts or by facilitating the mobility of scientists, who can then extend their social networks and become involved in collaborative research (Clark and Llorens, 2012; Bozeman and Corley, 2004). In support of this reasoning, we observed a positive association between funding rate and number of institutions in the CARD domain, where funding rates are lowest for non-collaborative papers (and where papers written as a by-product of clinical practice probably tend to concentrate), and highest for highly collaborative papers (among which clinical trial reports are probably frequent). The higher funding rate for highly collaborative papers is consistent with the implementation of different funding instruments in the health sciences in Spain (MINECO, 2013) aimed at fostering collaboration, particularly through initiatives designed to support thematic research networks (e.g. CIBER) (http://www.ciberisciii.es). Collaboration between clinicians and researchers, on the one hand, and between hospitals, academia and industry, on the other, is promoted to enhance translational research, i.e., to transfer scientific knowledge from basic research to clinical practice (Rey-Rocha and López-Navarro, 2014) with the ultimate aim of obtaining health benefits for patients throughout the country.

Contrary to what we report here for CARD, it is single-institution papers which attain the highest funding rate in VIROL (Figure 3, Table 3). This was an unexpected result, although it should be noted that differences in funding rates according to the number of institutions are small in this domain because the funding rate is above $80 \%$ regardless of the number of institutions. What emerges here is the lower influence of the number of institutions on the likelihood that authors in the VIROL domain will report funding, perhaps because the positive effect of interinstitutional collaboration on research is not as strong as in CARD. A possible reason for this difference between domains is that VIROL is an essentially basic science field, and "collaboration is believed to be more effective in fields with clear questions and less clear objectives do exist in basic sciences" (Toomela, 2007). Single-institution papers in VIROL reflect a very basic level (more basic than papers with authors from more institutions), with an above average involvement of government centres (basic orientation) and below-average involvement of hospitals. In any case it should be recalled that the outputs of a given grant comprise both single- and multi-institution papers, and that a single-institution paper may be one of the many results of a collaborative research project.

\section{Science policy implications}

The present study shows that an analysis of funding acknowledgements in publications provides information about the share of funded publications and funding patterns across disciplines, information that can be useful by research managers, policy makers and funding agencies. In particular, issues such as the share of public vs. private funding sources, the involvement of the business sector in supporting research, and the extent to which researchers benefit from international funding sources are currently questions of strategic interest from a research policy point of view. However, caution is needed when making comparisons between fields, because different factors such as the type of research (clinical vs. basic) and the authors' institutional setting may entail differences in the share of internal or external funding and the degree of reliance on competitive projects; consequently, these factors may be associated with different funding rates and patterns. Anyway, the development 
of follow-up studies can be useful to detect changes over time, for example in response to the implementation of specific research policy measures.

For the two domains analysed here, the positive association we observed between funding acknowledgement in the WOS FA field and variables for impact and international collaboration suggests that funders have achieved their objectives of supporting research of higher quality and favouring collaboration with foreign partners. Moreover, the greater influence of Q1 compared to citations indicates that funded researchers are aware of the need to disseminate their research through high-quality channels, even though they are not always rewarded with high citation rates. In this sense, it is worth noting that publication of research results in high impact factor journals is encouraged by the Spanish Programme for R\&D\&I (MINECO, 2013), which is the main research granting source in Spain, even in calls devoted to respond to societal challenges.

Because the names of funding agencies are not normalised in funding acknowledgements, much data-cleaning work was necessary to identify all agencies correctly in order to obtain reliable results. To reduce data-cleaning efforts and increase the reliability of future studies, policies will be needed to ensure that authors appropriately disclose funding sources in publications. Also desirable are further efforts to enhance the standardisation of funding data collected in bibliographic databases.

\section{Limitations and further research}

Some limitations of this study should be noted. Firstly, funded research might be underestimated in our study since authors may fail to acknowledge all sources of funds received. However, failure to acknowledge sources of support in published reports is increasingly rare now that reporting funding sources in publications is becoming mandatory in Spain. Secondly, we assume in this study that work reported in papers with no funding information received no specific funding, although we are aware that the work may have been supported by internal core funding, which is usually not acknowledged. However, our focus here is on external funding, usually allocated through a competitive process, since it may be more likely to have a positive impact on research (Begin-Caouette et al. 2017) ${ }^{1}$. Thirdly, nonEnglish-language papers are not considered in this study because funding acknowledgments are not covered by WoS for these publications, so we do not know to what extent our results apply to that set of papers. Finally, it should be noted that our findings cannot be generalized to other fields or countries.

Finally, because explicit acknowledgment of funding is increasingly required by funding organizations and scientific journals, an upward trend in the presence of FA in publications is expected in the years to come. Concurrently, our interest needs to focus on more specific aspects such as the type of funding instrument acknowledged (research projects, fellowships, infrastructures, etc.) and an analysis of co-funding and its effects on research, given that both of these aspects may differ across domains. Moreover, studies that go beyond an analysis of aggregate data to study the effects of funding at the individual level may provide more

\footnotetext{
${ }^{1}$ In relation to external funding, we can distinguish between competitive research funding, where the allocation of funds is usually based on peer review, and contract research funding, which is more applied, mission-oriented and less focused on research excellence. Public funding is mainly allocated through competitive mechanisms in OECD countries, while contract funding is more often in line with private sector objectives. Since public funding predominates in our sample (in 62\% of CARD papers and $91 \%$ of the VIROL ones), we assume that competitive allocation of funds predominates in our study.
} 
accurate and detailed data that can shed light on how funding contributes to research performance.

Acknowledgments. We are grateful to the Ministry of Economy and Competitiveness (MINECO) for funding research project CSO2014-57826-P, the pre-doctoral contract BES-2015073537 awarded to B.A.B., and the Juan de la Cierva-Formación Postdoctoral Training grant awarded to A.A.D.F. We would like to thank the ACUTE team for its support with data processing, and Carmen Cabanillas for developing programs to manage funding data. We are also grateful to Rodrigo Costas for suggestions and to Kevin Boyack for kindly providing the data on research levels. Appreciation is expressed to K. Shashok for improving the use of English in the manuscript.

\section{References}

Álvarez-Bornstein, B., Morillo, F. \& Bordons, M. (2017). Funding acknowledgements in the Web of Science: Completeness and accuracy of collected data. Scientometrics, 112 (3), 17931812.

Aström, f., Hedenfalk, I., Graffner, M. \& Nilbert, M. (2013). Effects of research funding, gender and type of position on research collaboration networks: a micro-level study of cancer research at Lund University. In: Gorraiz, J. et al (Eds.), Proceedings of the 14th International-Society-of-Scientometrics-and-Informetrics Conference (ISSI). Vienna, Austria: Austrian Institute of Technology.

Beaudry, C. \& Allaoui, S. (2012). Impact of public and private research funding on scientific production: the case of nanotechnology. Research Policy, 41(9), 1589-1606.

Begin-Caouette, O., Kalpazidou Schimidt, E. \& Field, C.C. (2017). The perceived impact of four funding streams on academic research production in Nordic countries: the perspectives of system actors. Science and Public Policy, 44(6), 789-801.

Bol, T., De Vaan, M.\& Van de Rijt (2018). The Matthew effect in science funding. PNAS, 115(19), 4887-4890.

Bordons, M., Aparicio, J. \& Costas, R. (2013). Heterogeneity of collaboration and its relationship with research impact in a biomedical field. Scientometrics, 96, 443-466.

Boyack, K.W. \& Jordan, P. (2011). Metrics associated with NIH funding: a high-level view. Journal of the American Medical Informatics Association, 18, 423-431.

Boyack, K.W., Patek, M., Ungar, L.H., Yoon, P. \& Klavans, R. (2014). Classification of individual articles from all of science by research level. Journal of Informetrics, 8, 1-12.

Bozeman, B. \& Corley, E. (2004). Scientists' collaboration strategies: implications for scientific and technical human capital. Research Policy, 33, 599-616.

Butler, J., Tahhan, A.S., Georgiopoulou, V.V., Kelkar, A., Lee, M., Khan, B., Peterson, E., Fonarow, G.C., Kalogeropoulos, A.P. \& Gheorghiade, M. (2015). Trends in characteristics of cardiovascular clinical trials 2001-2012. American Heart Journal, 170(2), 263-272.

Campbell, D., Picard-Aitken, M., Côté, G., Caruso, J., Valentim, R., Edmonds, S. \& Archambault, E. (2010). Bibliometrics as a performance measurement tool for research evaluation: The case of research funded by the National Cancer Institute of Canada. American Journal of Evaluation, 31, 66-83.

Carayol, N. \& Matt, M. (2006). Individual and collective determinants of academic scientists' productivity. Information Economics and Policy, 18(1), 55-72.

Clark, B.Y. \& Llorens, J. (2012). Investments in scientific research: examining the funding threshold effects on scientific collaboration and variation by academic discipline. Policy Studies Journal, 40(4), 698-729. 
Costas, R., \& Leeuwen, T. N. (2012). Approaching the "reward triangle": General analysis of the presence of funding acknowledgments and "peer interactive communication" in scientific publications. Journal of the Association for Information Science and Technology, 63(8), 1647-1661.

Decker, R.S., Wimsalt, L., Trice, A.G. \& Konstan J.A. (2011). A profile of federal-grant administratative burden among federal demonstration partnership faculty. A Report of the Faculty Standing Committee of the Federal Demonstration Partnership. http://www.iscintelligence.com/archivos subidos/usfacultyburden 5.pdf

Defazio, D., Lockett, A. \& Wright, M. (2009). Funding incentives, collaborative dynamics and scientific productivity: Evidence from the EU framework program. Research Policy, 38, 293-305.

De Granda-Orive, J., Alonso-Arroyo, A., Garcia-Rio, F., López-Padilla, D.E., Solano-Reina, S., Jiménez-Ruiz, C.A. \& Aleixandre-Benavent, R. (2015). Global funding for papers of excellence on smoking, 2010-2014. Revista Panamericana de Salud Pública-Pan american Journal of Public Health, 38(5), 410-417.

Ebadi, A. \& Schiffauerova, A. (2016). How to boost scientific production? A statistical analysis of research funding and other influencing factors. Scientometrics 106, 1093-1116.

European Commission (2016). The 2016 EU Industrial R\&D Investment Scoreboard. Luxembourg: Publications Office of the European Union. Doi:10.2791/84632. http://iri.jrc.ec.europa.eu/scoreboard16.html

Fortin, J.M. \& Currie, D.J (2013). Big science vs. little science: how scientific impact scale with funding. PLOS ONE 8(6): e65263. DOI:10.1371/journal.pone.0065263.

Fundación Cotec para la Innovación (2018). Informe Cotec 2018. http://informecotec.es/media/Informe-Cotec 2018 versi\%C3\%B3nweb.pdf

Godin, B. (2003). The impact of research grants on the productivity and quality of scientific research. INRS Working paper.

Gök, A., Rigby, J. \& Shapira, P. (2016). The impact of research funding on scientific outputs: Evidence from six smaller European countries. Journal of the Association for Information Science and Technology, 67, 715-730.

Goldfinch, S., Dale, T., \& DeRouen, K. (2003). Science from the periphery: Publication, collaboration and "periphery effects" in article citation rates of the New Zealand Crown Research Institutes 1995-2000. Scientometrics, 57(3), 321-337.

Harter, S.P. \& Hooten, P.A. (1990). Factors affecting funding and citation rates in information science publications. Library and Information Science Research, 10, 263-280.

Haslam, N., Ban, L. , Kaufmann, L., Loughnan, S., Peters, K., Whelan, J. \& Wilson, S. (2008). What makes an article influential? Predicting impact in social and personality psychology. Scientometrics, 76(1), 169-185.

Heffner, A.G. (1981). Funded research, multiple authorship and subauthorship collaboration in four disciplines. Scientometrics, 3(1), 5-12.

Hottenrott, H. \& Lawson, C. (2017). Fishing for complementarities: Research grants and research productivity. International Journal of Industrial Organization 51, 1-38.

International Committee of Medical Journal Editors [www.icmje.org] (2017). Recommendations for the Conduct, Reporting, Editing and Publication of Scholarly Work in Medical Journals [accessed on June/08/2018] Available from: http://www.ICMJE.org.

Jacob, B.A. \& Lefgren, L. (2011). The impact of research grant funding on scientific productivity. Journal of public economics, 95(9-10), 1168-1177.

Katz, J.S. \& Martin, B.R. (1997). What is research collaboration? Research Policy, 26 (1), 1-18.

Koier, E. \& Horlings, E. (2014). How accurately does output reflect the nature and design of transdisciplinary research programmes? Research Evaluation, 24, 37-50.

Langfeldt, L., Bloch, C.W. \& Sivertsen, G. (2015). Options and limitations in measuring the impact of research grants- evidence from Denmark and Norway. Research Evaluation, 24, 256-270. 
Lariviere, V., Gingras, Y., Sugimoto, C.R. \& Tsou, A. (2015). Team size matters: Collaboration and scientific impact since 1900. Journal of the Association for Information Science and Technology, 66 (7), 1323-1332.

Lee, S. \& Bozeman, B. (2005). The impact of research collaboration on scientific productivity. Social Studies of Science, 35(5), 673-702.

Lewison, G. (2003). The publication of cancer research papers in high impact journals. Aslib Proceedings, 55, (5-6), 379-387.

Lewison, G. \& Dawson, G. (1998). The effect of funding on the outputs of biomedical research. Scientometrics, 41(1-2), 17-27.

MINECO, 2013. Spanish Plan for Scientific and Technical Research and Innovation. 2013-2016. Ministerio de Economía, Industria y Competitividad. http://www.ciencia.gob.es/stfIs/MICINN/Investigacion/FICHEROS/Spanish RDTI Plan 20 13-2016.pdf

Müller, R. (2012). Collaborating in life science research groups: The question of authorship. Higher Education Policy, 25(3), 289-311.

Narin, F., Pinski, G., \& Gee, H. H. (1976). Structure of the biomedical literature. Journal of the American Society for Information Science, 27(1), 25-45.

Neufeld, J. (2016). Determining effects of individual research grants on publication ouput and impact: The case of the Emmy Noether Programme (German Research Foundation). Research Evaluation, 25(1), 50-61.

Pao, M.L. (1991). On the relationship of funding and research publications. Scientometrics, 20(1), 257-281.

Peritz, B.C. (1990). The citation impact of funded an unfunded research in economics. Scientometrics, 19(3-4), 199-206.

Puerta, J.L., Martin-Moreno, J.M., Bravo, S. \& Gutiérrez-Fuentes, J.A. (2011). Evaluation of the research performed in Spanish hospitals. Revista Clínica Española, 211(4), 169-178.

Reagans, R., \& Zuckerman, E. W. (2001). Diversity and productivity: the social capital of corporate R\&D teams. Organization Science, 12 (4), 502-517.

Rey-Rocha, J. \& López-Navarro, I. (2014). The fourth mission of hospitals and the role of researchers as innovation drivers in the public healthcare sector. Revista Española de Documentación Científica, 37(1), e028.

Rigby, J. (2011). Systematic grant and funding body acknowledgement data for publications: new dimensions and new controversies for research policy and evaluation. Research Evaluation, 20, 365-375.

Sandström, U (2009). Research quality and diversity of funding: A model for relating research money to output of research. Scientometrics, 79, 341-349.

Schneider, J. \& van Leeuwen, T.N. (2014). Analysing robustness and uncertainty levels of bibliometric performance statistics supporting science policy. A case study evaluating Danish postdoctoral funding. Research Evaluation, 23(4), 285-297.

Toomela, A. (2007). Sometimes one is more than two: When collaboration inhibits knowledge construction. Integrative psychological and behavioral science, 41(2), 198-207.

Ubfal, D. \& Maffioli, A. (2011). The impact of funding on research collaboration: Evidence from a developing country. Research Policy, 40, 1269-1279.

Wang, J., \& Shapira, P. (2011). Funding acknowledgement analysis: an enhanced tool to investigate research sponsorship impacts: the case of nanotechnology. Scientometrics, 87, 563-586.

Wang J, \& Shapira, P. (2015). Is there a relationship between research sponsorship and publication impact? An analysis of funding acknowledgments in nanotechnology papers. PLOS ONE, 10(2): e0117727.

Wuchty, S., Jones, B.F. \& Uzzi, B. (2007). The Increasing Dominance of Teams in Production of Knowledge. Science, 316, 1036-1039. 
Yan, E.J., Wu, C.J. \& Song, M. The funding factor: a cross-disciplinary examination of the association between research funding and citation impact (2018). Scientometrics 115, 1, 369-384.

Yegros-Yegros, A. \& Costas, R. (2013). Analysis of the Web of Science funding acknowledgement information for the design of indicators on external funding attraction. Proceedings of the $14^{\text {th }}$ Conference of the International Society of Scientometrics and Informetrics, 84-95.

Zhao, D. (2010). Characteristics and impact of grant-funded research: a case study of the library and information science field. Scientometrics, 84, 293-306. 
Appendix 1. Collaboration and impact indicators by domain and funding presence

\begin{tabular}{|c|c|c|c|c|c|c|}
\hline & \multirow{2}{*}{ CARD } & \multirow{2}{*}{ VIROL } & \multicolumn{2}{|c|}{ CARD } & \multicolumn{2}{|c|}{ VIROL } \\
\hline & & & $\begin{array}{l}\text { With } \\
\text { FA }\end{array}$ & $\begin{array}{c}\text { Without } \\
\text { FA }\end{array}$ & $\begin{array}{l}\text { With } \\
\text { FA }\end{array}$ & $\begin{array}{c}\text { Without } \\
\text { FA }\end{array}$ \\
\hline No. articles $^{a}$ & 2,752 & 1,263 & 1,572 & 1,180 & 1,136 & 127 \\
\hline Research level & 1.9 & 3.1 & 2.1 & 1.5 & 3.1 & 2.7 \\
\hline \multicolumn{7}{|l|}{ Collaboration } \\
\hline No. authors/article & 9.7 & 8.5 & 11.0 & 8.0 & 8.6 & 7.1 \\
\hline No. institutions/article & 5.9 & 4.9 & 7.0 & 4.5 & 4.9 & 4.3 \\
\hline$\%$ single-institution papers & 14.3 & 13.7 & 6.8 & 24.2 & 14.3 & 7.9 \\
\hline$\%$ collaboration (>1 inst.) & 85.7 & 86.3 & 93.2 & 75.8 & 85.7 & 92.1 \\
\hline$\%$ national collaboration $^{\mathrm{b}}$ & 55.7 & 57.4 & 59.0 & 51.2 & 56.5 & 65.4 \\
\hline$\%$ int. collaboration ${ }^{b}$ & 49.1 & 49.2 & 59.2 & 35.8 & 50.4 & 38.6 \\
\hline \multicolumn{7}{|l|}{ Journal publication } \\
\hline \% Articles in Q1 & 47.6 & 47.7 & 56.6 & 35.0 & 50.0 & 26.4 \\
\hline$\%$ Articles in D1 & 22.3 & 13.3 & 29.5 & 12.3 & 14.2 & 5.0 \\
\hline \multicolumn{7}{|l|}{ Citations } \\
\hline Citations/article (3 years) & 11.1 & 8.9 & 13.4 & 8.0 & 9.1 & 7.3 \\
\hline $\mathrm{RCR}$ & 1.4 & 1.1 & 1.7 & 1.1 & 1.1 & 0.9 \\
\hline$\%$ HCP10 & 18.7 & 12.7 & 23.7 & 12.1 & 13.0 & 10.2 \\
\hline
\end{tabular}

Notes: $\mathrm{RCR}=$ Relative citation rate. $\mathrm{HCP}=$ Highly cited paper

${ }^{\mathrm{a}}$ Citable items

${ }^{\mathrm{b}}$ Papers with both national and international collaboration are considered in both categories. 
Appendix 2. Results of logistic regression to explore contribution of public/private funding to high impact research

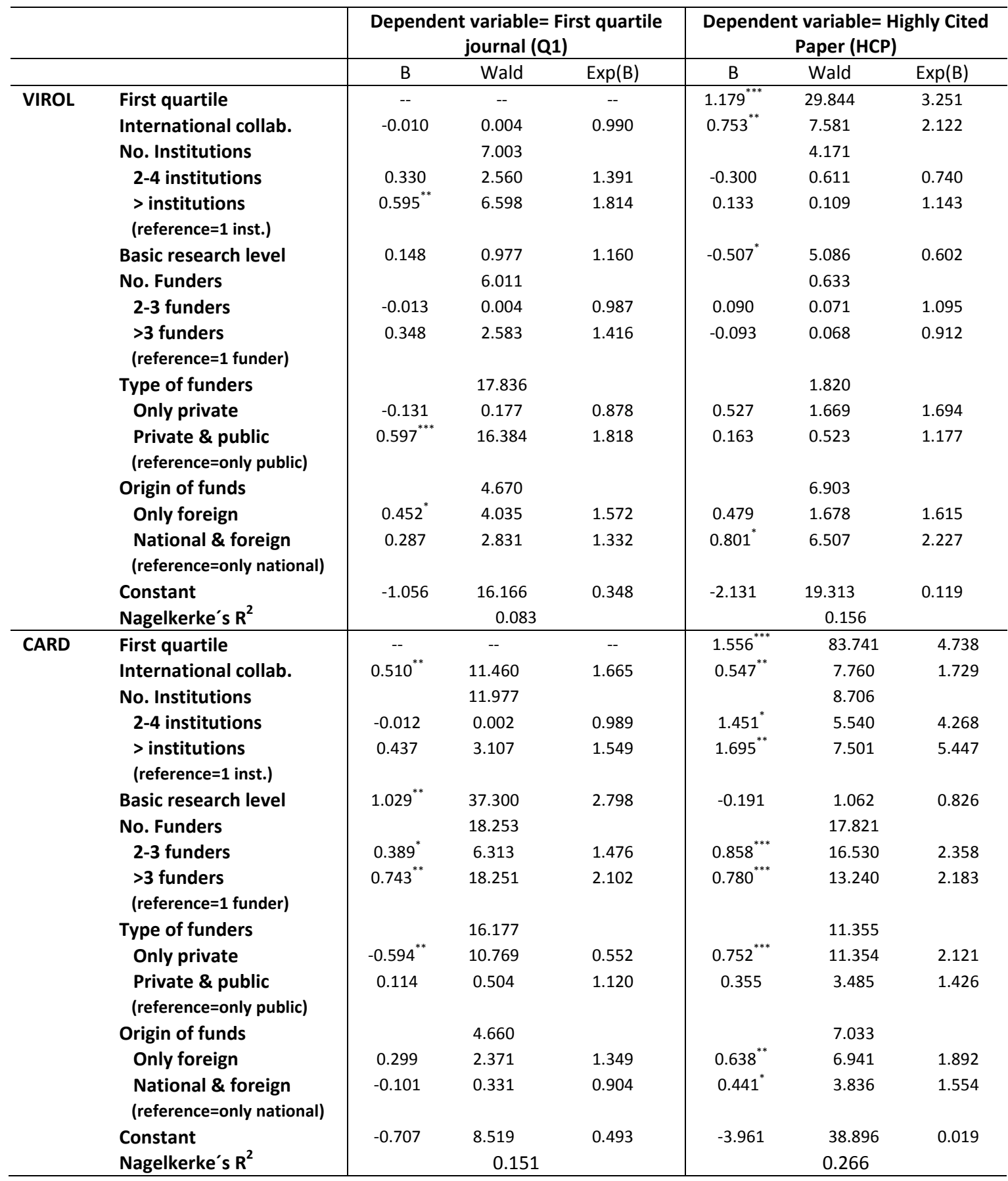

$* P<0.05 ; * * P<0.01 ; * * * P<0.001$

$\mathrm{OR}=$ Odds ratio $=\operatorname{Exp}(\mathrm{B})$

Note: the reference category was the first one for all variables. 